Pacific

Journal of

Mathematics

\title{
ON THE UNIFORM SQUEEZING PROPERTY OF BOUNDED CONVEX DOMAINS IN $\mathbb{C}^{n}$
}

\author{
KANG-TAE KIM AND LIYOU ZHANG
}




\title{
ON THE UNIFORM SQUEEZING PROPERTY OF BOUNDED CONVEX DOMAINS IN $\mathbb{C}^{n}$
}

\author{
KANG-TAE KIM AND LiYOU ZHANG
}

\begin{abstract}
We prove that the bounded convex domains and the $C^{2}$-smoothly bounded strongly pseudoconvex domains in $\mathbb{C}^{n}$ admit the uniform squeezing property. Moreover, we prove by the scaling method that the squeezing function approaches 1 near the strongly pseudoconvex boundary points.
\end{abstract}

\section{Introduction}

The notion of holomorphic homogeneous regular, or equivalently, uniformly squeezing for complex manifolds has been introduced in [Liu et al. 2004; 2005] and [Yeung 2009], respectively. This concept is essential for the estimation of several invariant metrics. See the above cited papers for details.

Let $\Omega$ be a complex manifold of dimension $n$. The squeezing function $\sigma_{\Omega}: \Omega \rightarrow \mathbb{R}$ of $\Omega$ is defined in [Deng et al. 2012] as follows. For each $p \in \Omega$, let

$$
\mathcal{F}(p, \Omega):=\left\{f: \Omega \rightarrow \mathbb{B}^{n}: f \text { is 1-1 holomorphic, } f(p)=0\right\},
$$

where

- $\mathbb{B}^{n}(p ; r)=\left\{z \in \mathbb{C}^{n}:\|z-p\|<r\right\}$, and

- $\mathbb{B}^{n}=\mathbb{B}^{n}(\mathbf{0} ; 1)=\mathbb{B}^{n}((0, \ldots, 0) ; 1)$.

Then define

$$
\sigma_{\Omega}(p)=\sup \left\{r: \mathbb{B}^{n}(\mathbf{0} ; r) \subset f(\Omega) \text { for some } f \in \mathcal{F}(p, \Omega)\right\} .
$$

Furthermore, the squeezing constant $\hat{\sigma}_{\Omega}$ for $\Omega$ is defined by

$$
\hat{\sigma}_{\Omega}:=\inf _{p \in \Omega} \sigma_{\Omega}(p) .
$$

Definition ([Liu et al. 2004; 2005; Yeung 2009]). A complex manifold $\Omega$ is called holomorphic homogeneous regular (HHR), or equivalently uniformly squeezing, if $\hat{\sigma}_{\Omega}>0$. 
Notice that the HHR property is preserved by biholomorphisms. The squeezing function and squeezing constants are also biholomorphic invariants [Deng et al. 2012].

These concepts have been developed for the study of completeness and other geometric properties such as the metric equivalence of the invariant metrics, including the Carathéodory, Kobayashi-Royden, Teichmüller, Bergman, and KählerEinstein metrics. It is obvious that the examples of HHR manifolds include bounded homogeneous domains. In case the manifold is biholomorphic to a bounded domain and the holomorphic automorphism orbits accumulate at every boundary point, such as in the case of the Bers embedding of the Teichmüller space, again the HHR property holds. A somewhat less obvious example are the bounded strongly convex domains (as the majority of them do not possess any holomorphic automorphisms except the identity map), proved by S.-K. Yeung [2009]. But there, some of the most standard examples, such as the bounded convex domains and the bounded strongly pseudoconvex domains, were left untouched.

Indeed, the starting point of this article is to show:

Theorem 1.1. All bounded convex domains in $\mathbb{C}^{n}(n \geq 1)$ are HHR.

Note that we do not assume any additional conditions such as boundary smoothness or "finite type" in the sense of D' Angelo in the above theorem. Nevertheless, the concept of squeezing function $\sigma_{\Omega}$ defined above plays an important role, and moreover, it appeals to us that further investigations of this function would be worthwhile. One immediate observation is that if $\sigma_{\Omega}(p)=1$ for some $p \in \Omega$, then $\Omega$ is biholomorphic to the unit open ball. In light of studies on the asymptotic behavior of several invariant metrics of strongly pseudoconvex domains, perhaps it is natural to ask, for a bounded strongly pseudoconvex domain $\Omega$ in $\mathbb{C}^{n}$, whether $\lim _{\Omega \ni q \rightarrow p} \sigma_{\Omega}(q)=1$ holds for every boundary point $p \in \partial \Omega$.

It was proved in [Deng et al. 2015] that the HHR property holds for all bounded strongly pseudoconvex domains, using an improvement of the method in [Fridman and Ma 1995]. In the present paper, by using a different approach — the scaling method - we will prove:

Theorem 1.2. If $\Omega$ is a bounded domain in $\mathbb{C}^{n}$ with a $C^{2}$ strongly convex boundary, then $\lim _{\Omega \ni q \rightarrow p} \sigma_{\Omega}(q)=1$ for every $p \in \partial \Omega$.

Actually, we have a more general conclusion in Theorem 3.1, which implies Theorem 1.2. The question posed above follows quickly from Theorem 3.1 and the following remarkable theorem of Diederich, Fornaess, and Wold [2014].

Theorem 1.3 [Diederich et al. 2014, Theorem 1.1]. Let $\Omega \subset \mathbb{C}^{n}$ be a bounded domain which is locally convexifiable and has finite type $2 k$ near a point $p \in \partial \Omega$. Assume further that $\partial \Omega$ is $C^{\infty}$-smooth near $p$, and that $\bar{\Omega}$ has a Stein neighborhood 
basis. Then there exists a holomorphic embedding $f: \bar{\Omega} \rightarrow \bar{B}_{k}^{n}$, where

$$
B_{k}^{n}=\left\{z \in \mathbb{C}^{2}:\left|z_{n}\right|^{2}+\left|z^{\prime}\right|^{2 k}<1\right\},
$$

such that $f(p)=(0, \ldots, 0,1)$ and $\left\{z \in \bar{\Omega}: f(z) \in \partial B_{k}^{n}\right\}=\{p\}$.

In particular, if $\partial \Omega$ is strongly pseudoconvex near $p(i . e ., k=1)$, it is enough to assume that $\partial \Omega$ is $C^{2}$-smooth near $p$.

We mention here that the proof of Theorem 1.2 is of interest in its own right, and also clarifies and simplifies some previously known theorems. These are mentioned in the final section.

\section{Bounded convex domains are HHR manifolds}

The aim of this section is to establish Theorem 1.1 stated above. Not only does this theorem cover the case left untreated in [Yeung 2009], but our method is different (see also [Deng et al. 2012] on this matter). Our method uses a version of the "scaling method in several complex variables" initiated by S. Pinchuk [1991]. In fact, we use the version presented in [Kim 1992], modified for the purpose of studying the asymptotic boundary behavior of holomorphic invariants.

Proof of Theorem 1.1. We proceed in 5 steps.

Step 1. Setup. Let $\Omega$ be a bounded convex domain in $\mathbb{C}^{n}$. Suppose that $\Omega$ is not HHR. Then there exists a sequence $\left\{q_{j}\right\}$ in $\Omega$ converging to a boundary point, say $q \in \partial \Omega$, such that

$$
\lim _{j \rightarrow \infty} \sigma_{\Omega}\left(q_{j}\right)=0 .
$$

Needless to say, it suffices to show that such a sequence cannot exist.

Step 2. The $j$-th orthonormal frame. Let $\langle$,$\rangle represent the standard Hermitian$ inner product of $\mathbb{C}^{n}$, and let $\|v\|=\sqrt{\langle v, v\rangle}$. For every $q \in \mathbb{C}^{n}$ and complex linear subspace $V$ of $\mathbb{C}^{n}$, denote by

$$
B^{V}(q, r)=\left\{p \in \mathbb{C}^{n}: p-q \in V \text { and }\|p-q\|<r\right\} .
$$

Now let $q \in \Omega$ and define the positive number $\lambda(q, V)$ by

$$
\lambda(q, V)=\max \left\{r>0: B^{V}(q, r) \subset \Omega\right\} .
$$

This number is finite for each $(q, V)$, whenever $\operatorname{dim} V>0$, since $\Omega$ is Kobayashi hyperbolic.

Fix the index $j$ momentarily. Then we choose an orthonormal basis for $\mathbb{C}^{n}$, with respect to the standard Hermitian inner product $\langle$,$\rangle . First consider$

$$
\lambda_{j}^{1}:=\lambda\left(q_{j}, \mathbb{C}^{n}\right) .
$$


There exists $q_{j}^{1 *} \in \partial \Omega$ such that $\left\|q_{j}^{1 *}-q_{j}\right\|=\lambda_{j}^{1}$. Let

$$
e_{j}^{1}=\frac{q_{j}^{1 *}-q_{j}}{\left\|q_{j}^{1 *}-q_{j}\right\|} .
$$

Then consider the complex span $\operatorname{Span}_{\mathbb{C}}\left\{e_{j}^{1}\right\}$, and let $V^{1}$ be its orthogonal complement in $\mathbb{C}^{n}$. Take

$$
\lambda_{j}^{2}:=\lambda\left(q_{j}, V^{1}\right)
$$

and $q_{j}^{2 *} \in \partial \Omega$ such that $q_{j}^{2 *}-q_{j} \in V^{1}$ and $\left\|q_{j}^{2 *}-q_{j}\right\|=\lambda_{j}^{2}$. Then let

$$
e_{j}^{2}:=\frac{q_{j}^{2 *}-q_{j}}{\left\|q_{j}^{2 *}-q_{j}\right\|} .
$$

With $e_{j}^{1}, e_{j}^{2}, \ldots, e_{j}^{\ell *}$ and $\lambda_{j}^{1}, \lambda_{j}^{2}, \ldots, \lambda_{j}^{\ell}$ chosen, the next elements $e_{j}^{\ell+1 *}$ and $\lambda_{j}^{\ell+1}$ are selected as follows. Denote by $V^{\ell}$ the complex orthogonal complement of $\operatorname{Span}_{\mathbb{C}}\left\{e_{j}^{1}, e_{j}^{2}, \ldots, e_{j}^{\ell}\right\}$. Then

$$
\lambda_{j}^{\ell+1}:=\lambda\left(q_{j}, V^{\ell}\right)
$$

and $q_{j}^{\ell+1 *} \in \partial \Omega$ are such that $q_{j}^{\ell+1 *}-q_{j} \in V^{\ell}$ and $\left\|q_{j}^{\ell+1 *}-q_{j}\right\|=\lambda_{j}^{\ell+1}$. Let

$$
e_{j}^{\ell+1}:=\frac{q_{j}^{\ell+1 *}-q_{j}}{\left\|q_{j}^{\ell+1 *}-q_{j}\right\|} .
$$

By induction, this process yields an orthonormal set $e_{j}^{1}, \ldots, e_{j}^{n}$ for $\mathbb{C}^{n}$ and the positive numbers $\lambda_{j}^{1}, \ldots, \lambda_{j}^{n}$.

Step 3. Stretching complex linear maps. Let $\hat{e}^{1}, \ldots, \hat{e}^{n}$ denote the standard orthonormal basis for $\mathbb{C}^{n}$, i.e.,

$$
\hat{e}^{1}=(1,0, \ldots, 0), \hat{e}^{2}=(0,1,0, \ldots, 0), \ldots, \hat{e}^{n}=(0, \ldots, 0,1) .
$$

Define the stretching linear map $L_{j}: \mathbb{C}^{n} \rightarrow \mathbb{C}^{n}$ by

$$
L_{j}(z)=\sum_{k=1}^{n} \frac{\left\langle z-q_{j}, e_{j}^{k}\right\rangle}{\lambda_{j}^{k}} \hat{e}^{k}
$$

for every $z \in \mathbb{C}^{n}$. Note that for each $j, L_{j}$ maps $\Omega$ biholomorphically onto its image.

Step 4. Supporting hyperplanes. Notice that

$$
L_{j}\left(q_{j}\right)=\mathbf{0}=(0, \ldots, 0), L_{j}\left(q_{j}^{1 *}\right)=\hat{e}^{1}, \ldots, L_{j}\left(q_{j}^{n *}\right)=\hat{e}^{n} .
$$

We shall consider the supporting hyperplanes, say $\Pi_{j}^{k}$ for $k=1, \ldots, n$, of $L_{j}(\Omega)$ at points $L_{j}\left(q_{j}^{k *}\right)$ for $k=1, \ldots, n$, respectively. 
Substep 4.1. The supporting hyperplane $\Pi_{j}^{1}$. As noted above, $L_{j}\left(q_{j}^{1 *}\right)=\hat{e}^{1}$. Due to the choice of $q_{j}^{1 *}$, the supporting hyperplane of $\Omega$ at $q_{j}^{1 *}$ must also support the sphere tangent to the boundary $\partial \Omega$. Consequently the supporting hyperplane $\Pi_{j}^{1}$ of $L_{j}(\Omega)$ must support a smooth surface (an ellipsoid) tangent to $L_{j}(\partial \Omega)$ at $\hat{e}^{1}$. Thus, the equation for this hyperplane $\Pi_{j}^{1}$ is

$$
\operatorname{Re}\left(z_{1}-1\right)=0
$$

(independently of $j$, being perpendicular to $\hat{e}^{1}$ ). We also note that

$$
L_{j}(\Omega) \subset\left\{\left(z_{1}, \ldots, z_{n}\right) \in \mathbb{C}^{n}: \operatorname{Re} z_{1}<1\right\} .
$$

Substep 4.2. The rest of the supporting hyperplanes $\Pi_{j}^{k}$, for $k \geq 2$. First consider the case $k=2$. Then the supporting hyperplane $\Pi_{j}^{2}$ passes through $L_{j}\left(q_{j}^{2 *}\right)=\hat{e}^{2}$. Since the restriction of $\Omega$ to $V^{1}$ contains the sphere in $V^{1}$ tangent to the restriction of $\partial \Omega$ at the point $\hat{e}^{2}$, the supporting hyperplane $\Pi_{j}^{2}$ restricted to $L_{j}\left(V^{1}\right)$ takes the equation $\left\{\left(z_{2}, \ldots, z_{n}\right) \in \mathbb{C}^{n}: \operatorname{Re}\left(z_{2}-1\right)=0\right\}$. Hence

$$
\Pi_{j}^{2}=\left\{\left(z_{1}, \ldots, z_{n}\right) \in \mathbb{C}^{n}: \operatorname{Re}\left(a_{j}^{2,1} z_{1}+a_{j}^{2,2}\left(z_{2}-1\right)\right)=0\right\}
$$

for some $\left(a_{j}^{2,1}, a_{j}^{2,1}\right) \in \mathbb{C}^{2}$ with $\left|a_{j}^{2,1}\right|^{2}+\left|a_{j}^{2,2}\right|^{2}=1$ and $a_{j}^{2,2}>0$. We also have that

$$
L_{j}(\Omega) \subset\left\{\left(z_{1}, \ldots, z_{n}\right) \in \mathbb{C}^{n}: \operatorname{Re}\left(a_{j}^{2,1} z_{1}+a_{j}^{2,2}\left(z_{2}-1\right)\right)<0\right\} .
$$

For $k \in\{3, \ldots, n\}$, one deduces inductively that the supporting hyperplane $\Pi_{j}^{k}$ passes through the point $\hat{e}^{k}$, and that

$$
\Pi_{j}^{k}=\left\{\left(z_{1}, \ldots, z_{n}\right) \in \mathbb{C}^{n}: \operatorname{Re}\left(a_{j}^{k, 1} z_{1}+\cdots+a_{j}^{k, k-1} z_{k-1}+a_{j}^{k, k}\left(z_{k}-1\right)\right)=0\right\},
$$

with $a_{j}^{k, k}>0$ and $\sum_{\ell=1}^{k}\left|a_{j}^{k, \ell}\right|^{2}=1$. Also,

$$
L_{j}(\Omega) \subset\left\{\left(z_{1}, \ldots, z_{n}\right) \in \mathbb{C}^{n}: \operatorname{Re}\left(a_{j}^{k, 1} z_{1}+\cdots+a_{j}^{k, k-1} z_{k-1}+a_{j}^{k, k}\left(z_{k}-1\right)\right)<0\right\} .
$$

Substep 4.3. Polygonal envelopes. We add this small substep for convenience. From the discussion so far in this step, we have the $j$-th polygonal envelope (of $L_{j}(\Omega)$ )

$$
\begin{aligned}
& \Sigma_{j}:=\left\{\left(z_{1}, \ldots, z_{n}\right) \in \mathbb{C}^{n}:\right. \operatorname{Re} z_{1}<1, \\
& \operatorname{Re}\left(a_{j}^{2,1} z_{1}+a_{j}^{2,2}\left(z_{2}-1\right)\right)<0, \\
& \vdots \\
& \\
&\left.\operatorname{Re}\left(a_{j}^{n, 1} z_{1}+\cdots+a_{j}^{n, n-1} z_{n-1}+a_{j}^{n, n}\left(z_{n}-1\right)\right)<0\right\} .
\end{aligned}
$$

Step 5. Bounded realization. Notice that, for every $k \in\{1, \ldots, n\}$, the disc

$$
D_{j}^{k}:=\left\{z=\left(z_{1}, \ldots, z_{n}\right) \in \mathbb{C}^{n}:\left\|z-q_{j}\right\|<\lambda_{j}^{k} \text { and } \forall \ell \neq k,\left\langle z-q_{j}, e_{j}^{\ell}\right\rangle=0\right\}
$$


is contained in $\Omega$. Hence, every $L_{j}(\Omega)$ contains the discs $D^{k}:=\left\{\zeta \hat{e}^{k}: \zeta \in \mathbb{C},|\zeta|<1\right\}$ for every $k=1, \ldots, n$. Since $\Omega$ is convex and $L_{j}$ is linear, $L_{j}(\Omega)$ is also convex. Therefore, the "unit acorn"

$$
A:=\left\{\left(z_{1}, \ldots, z_{n}\right) \in \mathbb{C}^{n}:\left|z_{1}\right|+\cdots+\left|z_{n}\right|<1\right\}
$$

is contained in $L_{j}(\Omega)$. This restricts the unit normal vectors

$$
n_{j}^{k}:=\left(a_{j}^{k, 1}, \ldots, a_{j}^{k, k}, 0, \ldots, 0\right) \in \mathbb{C}^{n}
$$

for every $k=2, \ldots, n$. Namely, there is a positive constant $\delta>0$ independent of $j$ and $k$ such that $a_{j}^{k, k} \geq \delta$ for every $j, k$.

Now taking a subsequence (of $q_{j}$ ), we may assume that the sequence of unit vectors $\left\{n_{j}^{k}\right\}_{j=1}^{\infty}$ converges for every $k \in\{2, \ldots, n\}$. Let us write

$$
\lim _{j \rightarrow \infty} n_{j}^{k}=n^{k}=\left(a^{k, 1}, \ldots, a^{k, k}, 0, \ldots, 0\right)
$$

for each $k=1,2, \ldots, n$.

Consider the maps

$$
B_{j}\left(z_{1}, \ldots, z_{n}\right)=\left(\zeta_{1}, \ldots, \zeta_{n}\right)
$$

defined by

$$
\zeta_{1}=z_{1}, \zeta_{2}=a_{j}^{2,1} z_{1}+a_{j}^{2,2} z_{2}, \ldots, \zeta_{n}=a_{j}^{n, 1} z_{1}+\cdots+a_{j}^{n, n} z_{n} .
$$

Then it follows that

$$
\begin{aligned}
B_{j} \circ L_{j}(\Omega) & \subset B_{j}\left(\Sigma_{j}\right) \\
& =\left\{\left(\zeta_{1}, \ldots, \zeta_{n}\right) \in \mathbb{C}^{n}: \operatorname{Re} \zeta_{1}<1, \operatorname{Re} \zeta_{2}<a_{j}^{2,2}, \ldots, \operatorname{Re} \zeta_{n}<a_{j}^{n, n}\right\} .
\end{aligned}
$$

Now, for each $j$, we consider the Cayley transformation

$$
\Phi_{j}\left(z_{1}, \ldots, z_{n}\right)=\left(\frac{z_{1}}{2-z_{1}}, \frac{z_{2}}{2 a_{j}^{2,2}-z_{2}}, \ldots, \frac{z_{n}}{2 a_{j}^{n, n}-z_{n}}\right) .
$$

Then $\Phi_{j} \circ B_{j}\left(\Sigma_{j}\right) \subset D^{n}$, where $D^{n}$ denotes the unit polydisc in $\mathbb{C}^{n}$ centered at the origin. Also, there exists a positive constant $\delta^{\prime} \in(0, \delta)$ such that $\Phi_{j} \circ B_{j}\left(\Sigma_{j}\right) \subset D^{n}$ contains the ball of radius $\delta^{\prime}$ centered at the origin $\mathbf{0}$.

Since $\Phi_{j} \circ B_{j} \circ L_{j}\left(q_{j}\right)=(0, \ldots, 0)$ for every $j$, we now conclude that the squeezing function satisfies

$$
\sigma_{\Omega}\left(q_{j}\right) \geq \frac{\delta^{\prime}}{\sqrt{n}}
$$

This estimate, which holds for every sequence $q_{j}$ approaching the boundary, yields the desired contradiction at last. Thus the proof is complete. 


\section{Boundary behavior of the squeezing function on strongly convex domains}

Definition. Let $\Omega$ be a domain in $\mathbb{C}^{n}$. A boundary point $p \in \partial \Omega$ is said to be spherically extreme if

(1) the boundary $\partial \Omega$ is $C^{2}$-smooth in an open neighborhood of $p$, and

(2) there exists a ball $\mathbb{B}^{n}(c(p) ; R)$ in $\mathbb{C}^{n}$ of some radius $R$, centered at some point $c(p)$ such that $\Omega \subset \mathbb{B}^{n}(c(p) ; R)$ and $p \in \partial \Omega \cap \partial \mathbb{B}^{n}(c(p) ; R)$.

The main goal of this section is to establish the following theorem.

Theorem 3.1. If a domain $\Omega$ in $\mathbb{C}^{n}$ admits a spherically extreme boundary point $p$ in a neighborhood of which the boundary $\partial \Omega$ is $C^{2}$-smooth, then

$$
\lim _{\Omega \ni q \rightarrow p} \sigma_{\Omega}(q)=1
$$

Since every boundary point of a $C^{2}$ strongly convex bounded domain is spherically extreme, this theorem implies Theorem 1.2. The rest of this section is devoted to the proof of Theorem 3.1.

Proof. The proof proceeds in seven steps.

Step 1. Sphere envelopes. Let $\Omega$ be a bounded domain in $\mathbb{C}^{n}$ with a boundary point $p \in \partial \Omega$ such that

(i) $\partial \Omega \cap \mathbb{B}^{n}\left(p ; r_{0}\right)$ is $C^{2}$-smooth for some $r_{0}>0$, and

(ii) $p$ is a spherically extreme boundary point of $\Omega$.

Then there exist positive constants $r_{1}, r_{2}$, and $R$ with $r_{0}>r_{1}>r_{2}$ such that every $q \in \Omega \cap \mathbb{B}^{n}\left(p ; r_{2}\right)$ admits points $b(q) \in \partial \Omega \cap \mathbb{B}^{n}\left(p ; r_{1}\right)$ and $c(q) \in \mathbb{C}^{n}$ satisfying the conditions

(iii) $\|q-b(q)\|<\|q-z\|$ for any $z \in \partial \Omega-\{b(q)\}$, and

(iv) $\|c(q)-b(q)\|=R$ and $\Omega \subset \mathbb{B}^{n}(c(q) ; R)$.

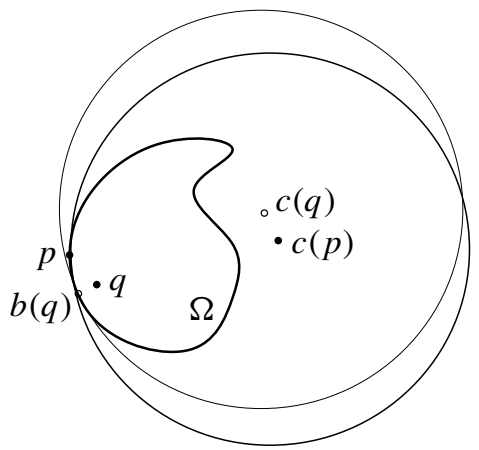

Figure 1. Sphere envelopes. 
See Figure 1. Notice that (iii) says that $b(q)$ is the unique boundary point that is the closest to $q$, and that the constant $R$ in (iv) is independent of the choice of $q \in \mathbb{B}^{n}\left(p ; r_{2}\right)$.

Step 2. Centering. In the following we shall use the familiar notation

$$
\begin{aligned}
z & =\left(z_{1}, \ldots, z_{n}\right), & z^{\prime} & =\left(z_{2}, \ldots, z_{n}\right), \\
u & =\operatorname{Re} z_{1}, & v & =\operatorname{Im} z_{1} .
\end{aligned}
$$

For each $q \in \Omega \cap \mathbb{B}^{n}\left(p ; r_{2}\right)$, choose a unitary transform $U_{q}$ of $\mathbb{C}^{n}$ such that the map $A_{q}(z):=U_{q}(z-b(q))$, depicted in Figure 2, satisfies the following conditions:

$$
A_{q}(q)=\left(\lambda_{q}, 0, \ldots, 0\right)
$$

for some $\lambda_{q}>0$, and

$$
A_{q}(\Omega) \subset \mathbb{B}^{n}((R, 0, \ldots, 0) ; R)=\left\{z \in \mathbb{C}^{n}:\left|z_{1}-R\right|^{2}+\left\|z^{\prime}\right\|^{2}<R^{2}\right\} .
$$

Then there exists a positive constant $r_{3}<r_{2}$ such that

$$
\begin{aligned}
z \in A_{q}(\Omega) & \cap \mathbb{B}^{n}\left(\mathbf{0} ; r_{3}\right) \\
& \Longleftrightarrow\|z\|<r_{3} \text { and } 2 u>H_{b(q)}\left(z^{\prime}\right)+\mathcal{K}_{b(q)}\left(v, z^{\prime}\right)+\mathcal{R}_{b(q)}\left(v, z^{\prime}\right),
\end{aligned}
$$

where

- $H_{b(q)}$ is a quadratic positive-definite Hermitian form such that there exists a constant $c_{0}>0$, independent of $q$, satisfying

$$
H_{b(q)}\left(z^{\prime}\right) \geq c_{0}\left\|z^{\prime}\right\|^{2},
$$

and

- there exists a constant $C>0$, independent of $q \in \mathbb{B}^{n}\left(p ; r_{3}\right) \cap \Omega$, such that

$$
\left|\mathcal{K}_{b(q)}\left(v, z^{\prime}\right)\right| \leq C\left(|v|^{2}+|v|\left\|z^{\prime}\right\|\right)
$$

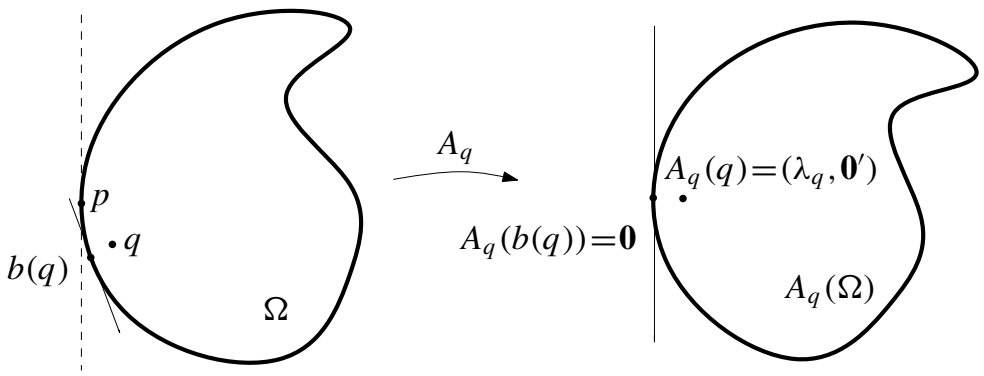

Figure 2. The centering process. 
whenever $z \in \mathbb{B}^{n}\left(\mathbf{0} ; r_{3}\right)$. Furthermore, we have

$$
\left|\mathcal{R}_{b(q)}\left(v, z^{\prime}\right)\right|=o\left(|v|^{2}+\left\|z^{\prime}\right\|^{2}\right) .
$$

In particular, the choice of $r_{3}$ can allow us the estimate

$$
\left|\mathcal{R}_{b(q)}\left(v, z^{\prime}\right)\right| \leq \frac{c_{0}}{2}\left(|v|^{2}+\left\|z^{\prime}\right\|^{2}\right) .
$$

Notice that

$$
\lim _{\Omega \ni q \rightarrow p} b(q)=p, \quad \lim _{\Omega \ni q \rightarrow p} H_{b(q)}\left(z^{\prime}\right)=H_{p}\left(z^{\prime}\right),
$$

and

$$
\lim _{\Omega \ni q \rightarrow p} A_{q}=I \text { (the identity map). }
$$

The latter limit and an inductive construction yield that for each integer $m>2$ there exists a strictly increasing integer-valued function $k(m)$ such that

$$
\mathbb{B}^{n}\left(\mathbf{0} ; r_{3} /(2 k(m))\right) \subset A_{q}\left(\mathbb{B}^{n}\left(p ; r_{3} / k(m)\right)\right) \subset \mathbb{B}^{n}\left(\mathbf{0} ; r_{3} / m\right)
$$

whenever $q \in \mathbb{B}^{n}\left(p ; r_{3} /(2 k(m))\right)$.

Step 3. The Cayley transform. The Cayley transform considered here is the map

$$
\kappa(z):=\left(\frac{1-z_{1}}{1+z_{1}}, \frac{\sqrt{2} z_{2}}{1+z_{1}}, \ldots, \frac{\sqrt{2} z_{n}}{1+z_{1}}\right),
$$

well-defined except at points of $Z=\left\{z \in \mathbb{C}^{n}: z_{1}=-1\right\}$. Notice that this transform maps the open unit ball $\mathbb{B}^{n}(\mathbf{0} ; 1)$ biholomorphically onto the Siegel half-space

$$
\mathcal{S}_{0}:=\left\{z \in \mathbb{C}^{n}: 2 \operatorname{Re} z_{1}>\left\|z^{\prime}\right\|^{2}\right\} .
$$

Moreover, $\kappa \circ \kappa=1$ and consequently, $\kappa\left(\mathcal{S}_{0}\right)=\mathbb{B}^{n}(\mathbf{0} ; 1)$. Notice also that, for $\mathbf{1}=(1,0, \ldots)$ and $-\mathbf{1}=(-1,0, \ldots)$, we have $\kappa(\mathbf{1})=(0, \ldots, 0), \kappa((0, \ldots, 0))=\mathbf{1}$, $\kappa(-\mathbf{1})=\infty$, and $\kappa(\infty)=-\mathbf{1}$.

Step 4. Stretching. Let $q \in \Omega \cap \mathbb{B}^{n}\left(p ; r_{3} /(2 k(m))\right)$. If we let $m$ tend to infinity, then of course $A_{q}(q)=\left(\lambda_{q}, 0, \ldots, 0\right)$ approaches $A_{q}(b(q))=(0, \ldots, 0)$, and so $\lambda_{q}$ approaches zero. For simplicity, we write $\lambda=\lambda_{q}$, suppressing the $q$ but keeping in mind that $\lambda$ is still dependent upon $q$. Note that

$$
A_{q}\left(\mathbb{B}^{n}(c(q) ; R)\right)=\left\{z \in \mathbb{C}^{n}: 2 R \cdot \operatorname{Re} z_{1}>\|z\|^{2}\right\} .
$$

Define the stretching map $\Lambda_{\lambda}: \mathbb{C}^{n} \rightarrow \mathbb{C}^{n}$, first introduced in [Pinchuk 1991], by

$$
\Lambda_{\lambda}(z):=\left(\frac{z_{1}}{\lambda}, \frac{z_{2}}{\sqrt{\lambda}}, \ldots, \frac{z_{n}}{\sqrt{\lambda}}\right) .
$$


Recall (3-6). The stretching map transforms $A_{q}(\Omega) \cap \mathbb{B}^{n}\left(\mathbf{0} ; r_{3} / 3\right)$ to the domain $\Lambda_{\lambda}\left(A_{q}(\Omega) \cap \mathbb{B}^{n}\left(\mathbf{0} ; r_{3} / 3\right)\right)$ so that

$$
\begin{aligned}
z \in \Lambda_{\lambda} \circ A_{q}(\Omega) & \cap \mathbb{B}^{n}\left(\mathbf{0} ; \frac{r_{3}}{\sqrt{\lambda} k(3)}\right) \\
\Longleftrightarrow\|z\| & <\frac{r_{3}}{\sqrt{\lambda} k(3)} \text { and } \\
2 u & >H_{b(q)}\left(z^{\prime}\right)+\frac{1}{\lambda} K_{b(q)}\left(\lambda v, \sqrt{\lambda} z^{\prime}\right)+\frac{1}{\lambda} \mathcal{R}_{b(q)}\left(\lambda v, \sqrt{\lambda} z^{\prime}\right) .
\end{aligned}
$$

On the other hand, notice that

$$
\left\|\frac{1}{\lambda} K_{b(q)}\left(\lambda v, \sqrt{\lambda} z^{\prime}\right)\right\| \leq C \sqrt{\lambda}\left(\sqrt{\lambda}|v|^{2}+|v|\left\|z^{\prime}\right\|\right)
$$

and that

$$
\left\|\frac{1}{\lambda} \mathcal{R}_{b(q)}\left(\lambda v, \sqrt{\lambda} z^{\prime}\right)\right\| \leq \frac{1}{\lambda} o\left(\left(|\lambda v|^{2}+\left\|\sqrt{\lambda} z^{\prime}\right\|^{2}\right)\right)=\frac{1}{\lambda} o(\lambda)
$$

on $\mathbb{B}^{n}(\mathbf{0} ; \rho)$ for any fixed constant $\rho>0$. Notice that both terms approach zero as $\lambda$ tends to zero. Thus, these terms can become sufficiently small if we limit $q$ to being contained in $\mathbb{B}^{n}\left(p ; r_{3} /(2 k(m))\right)$ for some sufficiently large $m$.

Step 5. Set-convergence. This step is in part heuristic; the heuristics appearing in this step, especially those which concern set convergences, are not used in the proof, strictly speaking. We include this step because they seem to help us to grasp the logical structure of the proof. On the other hand, the constructions in (3-13)-(3-15) shall be used in the remainder of the proof, especially in Step 7.

The main role of the stretching map $\Lambda_{\lambda}$, as $\lambda \searrow 0$, is to rescale the domains successively, letting them converge to the set limits.

For instance, if one considers

$$
\Lambda_{\lambda}\left(A_{q}(\Omega) \cap \mathbb{B}^{n}\left(\mathbf{0} ; r_{3}\right)\right),
$$

then one can see that $\Lambda_{\lambda}\left(\mathbb{B}^{n}\left(\mathbf{0} ; r_{3}\right)\right)$ contains $\mathbb{B}^{n}\left(\mathbf{0} ; r_{3} / \sqrt{\lambda}\right)$, a very large ball which exhausts $\mathbb{C}^{n}$ as $\lambda$ approaches zero. In the meantime, within that large ball, $\Lambda_{\lambda}\left(A_{q}(\Omega)\right)$ is restricted only by the inequality

$$
2 u>H_{b(q)}\left(z^{\prime}\right)+\widetilde{K}_{\lambda}\left(v, z^{\prime}\right),
$$

where $\widetilde{K}_{\lambda}=o(\lambda)$ is small enough to be negligible. One can imagine that indeed the "limit domain" of this procedure should be

$$
\widehat{\Omega}:=\left\{z \in \mathbb{C}^{n}: 2 u>H_{p}\left(z^{\prime}\right)\right\} .
$$

Here, of course, $H_{p}\left(z^{\prime}\right)$ is the quadratic positive-definite Hermitian form which appears in the defining inequality of $\Omega$ about the boundary point $p$ (understood as 
the origin):

$$
2 \operatorname{Re} z_{1}>H_{p}\left(z^{\prime}\right)+o\left(\left|\operatorname{Im} z_{1}\right|+\left\|z^{\prime}\right\|^{2}\right) .
$$

Notice that

$$
\kappa(\widehat{\Omega})=\left\{z \in \mathbb{C}^{n}:\left|z_{1}\right|^{2}+H_{p}\left(z^{\prime}\right)<1\right\},
$$

and hence there is a $\mathbb{C}$-linear isomorphism

$$
L: \mathbb{C}^{n} \rightarrow \mathbb{C}^{n}
$$

that maps $\kappa(\widehat{\Omega})$ biholomorphically onto the unit ball $\mathbb{B}^{n}(\mathbf{0} ; 1)$ with $L(\mathbf{1})=\mathbf{1}$.

Before moving on to the next step we remark that, since $\Omega \subset \mathbb{B}^{n}(c(q) ; R)$ whenever $q \in \mathbb{B}^{n}\left(p ; r_{2}\right)$,

$$
A_{q}(\Omega) \subset A_{q}\left(\mathbb{B}^{n}(c(q) ; R)\right)=\mathbb{B}^{n}((R, 0, \ldots, 0) ; R) .
$$

This in turn implies that

$$
\begin{aligned}
\Lambda_{\lambda} \circ A_{q}(\Omega) & \subset \Lambda_{\lambda}\left(\mathbb{B}^{n}((R, 0, \ldots, 0) ; R)\right) \\
& \subset \mathcal{E}:=\left\{z \in \mathbb{C}^{n}: 2 R \cdot \operatorname{Re} z_{1}>\left\|z^{\prime}\right\|^{2}\right\} .
\end{aligned}
$$

The last inclusion follows by (3-10).

Step 6. Auxiliary domains. Let $\delta>0$ be given. Consider the domains

$$
\begin{aligned}
\mathcal{G}_{\delta} & :=\left\{z \in \mathbb{C}^{n}: 2 u>-\delta|v|+(1-\delta) H_{b(q)}\left(z^{\prime}\right)\right\}, \\
\mathcal{F}_{\delta} & :=\left\{z \in \mathbb{C}^{n}: 2 u>\delta|v|+(1+\delta) H_{b(q)}\left(z^{\prime}\right)\right\}, \text { and } \\
\mathcal{H}_{q} & :=\left\{z \in \mathbb{C}^{n}: 2 u>H_{b(q)}\left(z^{\prime}\right)\right\},
\end{aligned}
$$

in addition to $\widehat{\Omega}$ and $\mathcal{E}$ introduced in (3-13) and (3-15).

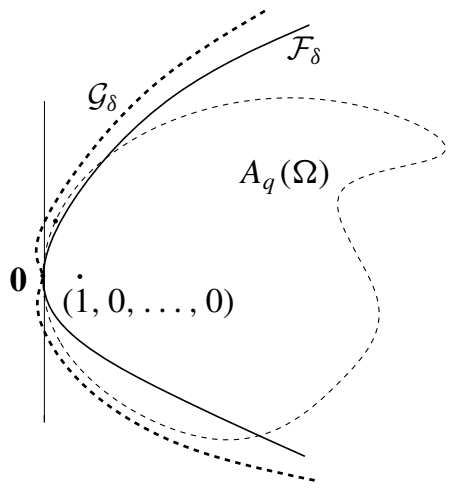

Figure 3. Auxiliary domains $\mathcal{G}_{\delta}$ and $\mathcal{F}_{\delta}$. 


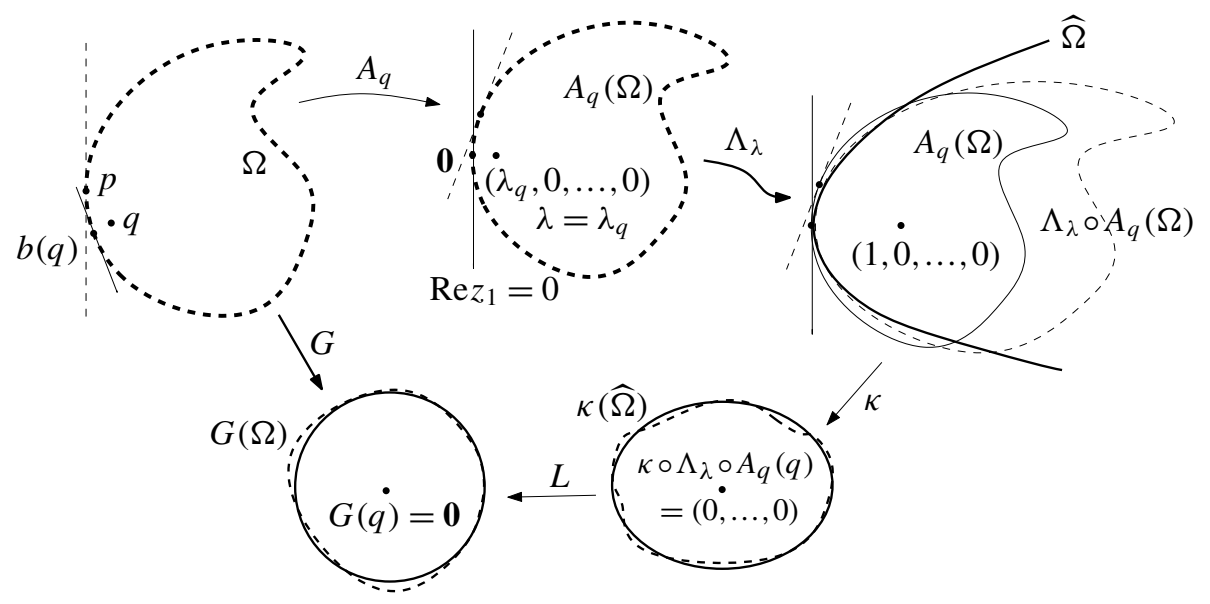

Figure 4. $G(\Omega)=L \circ \kappa \circ \Lambda_{\lambda} \circ A_{q}(\Omega)$ for $q \sim p$.

A straightforward computation checks that the image $\kappa\left(\mathcal{G}_{\delta}\right)$ of $\mathcal{G}_{\delta}$ via the Cayley transform $\kappa$ introduced earlier is

$$
\kappa\left(\mathcal{G}_{\delta}\right)=\left\{z \in \mathbb{C}^{n}:\left|z_{1}\right|^{2}-\frac{\delta}{2}\left|z_{1}-\bar{z}_{1}\right|+(1-\delta) H_{b(q)}\left(z^{\prime}\right)<1\right\} .
$$

Hence, there exists $\delta_{0}>0$ such that, for every $\delta$ with $0<\delta<\delta_{0}, \kappa\left(\mathcal{G}_{\delta}\right)$ is a bounded domain. Notice also that this domain is arbitrarily close to the domain $\kappa\left(\mathcal{H}_{b(q)}\right)$ as $\delta_{0}$ becomes arbitrarily small. It follows therefore that, for every $\epsilon>0$, there exists $\delta_{0}>0$ such that

$$
L \circ \kappa\left(\mathcal{G}_{\delta}\right) \subset \mathbb{B}^{n}(\mathbf{0} ; 1+\epsilon)
$$

whenever $0<\delta<\delta_{0}$. Moreover, observe that the stretching map $\Lambda_{\lambda}$ preserves all such domains as

$$
\mathcal{F}_{\delta}, \quad \mathcal{G}_{\delta}, \quad \mathcal{H}_{q}, \quad \widehat{\Omega}, \quad \text { and } \mathcal{E} .
$$

Let us now define the expression

$$
G(z):=L \circ \kappa \circ \Lambda_{\lambda} \circ A_{q}(z)
$$

for $z \in \mathbb{C}^{n}-\left(\Lambda_{\lambda} \circ A_{q}\right)^{-1}(Z)$. (The set $Z$ is defined in (3-8). Notice that this expression $G$ depends upon $q \in \mathbb{B}^{n}\left(\mathbf{0} ; r_{2}\right)$; see Figure 3 for an illustration.) In particular, this $G$ maps $\Omega$ onto its image $G(\Omega)$ biholomorphically. See also Figure 4.

Step 7. Proof of Theorem 3.1. Our final goal is to establish the following claim.

Claim. For any $\epsilon$ with $0<\epsilon<1 / 2$, there exists an integer $m>0$ such that

$$
\mathbb{B}^{n}(\mathbf{0} ; 1-\epsilon) \subset G(\Omega) \subset \mathbb{B}^{n}(\mathbf{0} ; 1+\epsilon)
$$

whenever $q \in \Omega \cap \mathbb{B}^{n}\left(p ; r_{3} /(2 k(m))\right)$. 
Since $G(q)=\mathbf{0}$, this implies that the squeezing function $\sigma_{\Omega}$ satisfies

$$
\sigma_{\Omega}(q) \geq \frac{1-\epsilon}{1+\epsilon} .
$$

Notice that this completes the proof of Theorem 3.1. Therefore it remains only to prove the claim.

Proof. Start with $\mathbb{B}^{n}(\mathbf{0} ; 1-\epsilon)$. Notice first, by the definition of $\mathcal{F}_{\delta}$, that for every $\delta>0$ there exists $m_{1}>0$ such that

$$
\mathcal{F}_{\delta} \cap \mathbb{B}^{n}\left(\mathbf{0} ; r_{2} / m\right) \subset A_{q}(\Omega) \cap \mathbb{B}^{n}\left(\mathbf{0} ; r_{2} / m\right)
$$

for any $m>m_{1}$.

Also,

$$
\kappa^{-1} \circ L^{-1}\left(\mathbb{B}^{n}(\mathbf{0} ; 1-\epsilon)\right) \Subset \kappa^{-1} \circ L^{-1}\left(\mathbb{B}^{n}(\mathbf{0} ; 1)\right)=\widehat{\Omega} .
$$

As discussed in (3-4)-(3-7), $L \circ \kappa\left(\mathcal{H}_{q}\right)$ is sufficiently close to $L \circ \kappa(\widehat{\Omega})$, which is the unit ball, whenever $q \in \mathbb{B}^{n}\left(p ; r_{3} /(2 k(m))\right)$ and $m$ is sufficiently large. Therefore there exists an integer $m_{2}>m_{1}$ such that $(L \circ \kappa)^{-1}\left(\mathbb{B}^{n}(\mathbf{0} ; 1-\epsilon)\right) \Subset \mathcal{H}_{q}$ whenever $q \in \mathbb{B}^{n}\left(p ; r_{3} / m_{2}\right)$.

As in (3-19), a direct computation yields

$$
\kappa\left(\mathcal{F}_{\delta}\right)=\left\{z \in \mathbb{C}^{n}:\left|z_{1}\right|^{2}+\frac{1}{2} \delta\left|z_{1}-\bar{z}_{1}\right|+(1+\delta) H_{b(q)}\left(z^{\prime}\right)<1\right\} .
$$

Now, consider the set $L \circ \kappa \circ \Lambda_{\lambda}\left(\mathcal{F}_{\delta}\right)$ for each $\delta>0$. (Recall that $\Lambda_{\lambda}\left(\mathcal{F}_{\delta}\right)=\mathcal{F}_{\delta}$ as remarked in the line below (3-20).) These domains increase monotonically as $\delta \searrow 0$ (since the $\mathcal{F}_{\delta}$ 's do) in such a way that the union $\bigcup_{0<\delta<\delta_{0}} L \circ \kappa \circ\left(\mathcal{F}_{\delta}\right)$ becomes arbitrarily close to $\mathbb{B}^{n}(\mathbf{0} ; 1)$ for $m$ sufficiently large. Consequently there exists a constant $\delta>0$ such that $\mathbb{B}^{n}(\mathbf{0} ; 1-\epsilon) \Subset L \circ \kappa \circ\left(\mathcal{F}_{\delta}\right)$. Moreover there is an integer $m_{3}>m_{2}$ such that

$$
\Lambda_{\lambda}^{-1}\left(\kappa^{-1} \circ L^{-1}\left(\mathbb{B}^{n}(\mathbf{0} ; 1-\epsilon)\right) \subset \mathbb{B}^{n}\left(\mathbf{0} ; r_{3} / k\left(m_{1}\right)\right),\right.
$$

as $\Lambda_{\lambda}^{-1}$ scales down the compact subsets (for $\lambda<r_{3} / m_{2}$ sufficiently small) to a small set near the origin. Hence, we have

$$
\Lambda_{\lambda}^{-1}\left(\kappa^{-1} \circ L^{-1}\left(\mathbb{B}^{n}(\mathbf{0} ; 1-\epsilon)\right)\right) \subset \mathcal{F}_{\delta} \cap \mathbb{B}^{n}\left(\mathbf{0} ; r_{3} / k\left(m_{1}\right)\right) \subset \Omega .
$$

Consequently, as long as $q \in \mathbb{B}^{n}\left(p ; r_{3} /\left(2 k\left(m_{3}\right)\right)\right)$,

$$
\begin{aligned}
\mathbb{B}^{n}(\mathbf{0} ; 1-\epsilon) & \subset L \circ \kappa \circ \Lambda_{\lambda}\left(\mathcal{F}_{\delta} \cap \mathbb{B}^{n}\left(\mathbf{0} ; r_{3} / k\left(m_{1}\right)\right)\right) \\
& \subset L \circ \kappa \circ \Lambda_{\lambda}\left(A_{q}(\Omega)\right) \\
& =G(\Omega) .
\end{aligned}
$$

See Figure 5. 


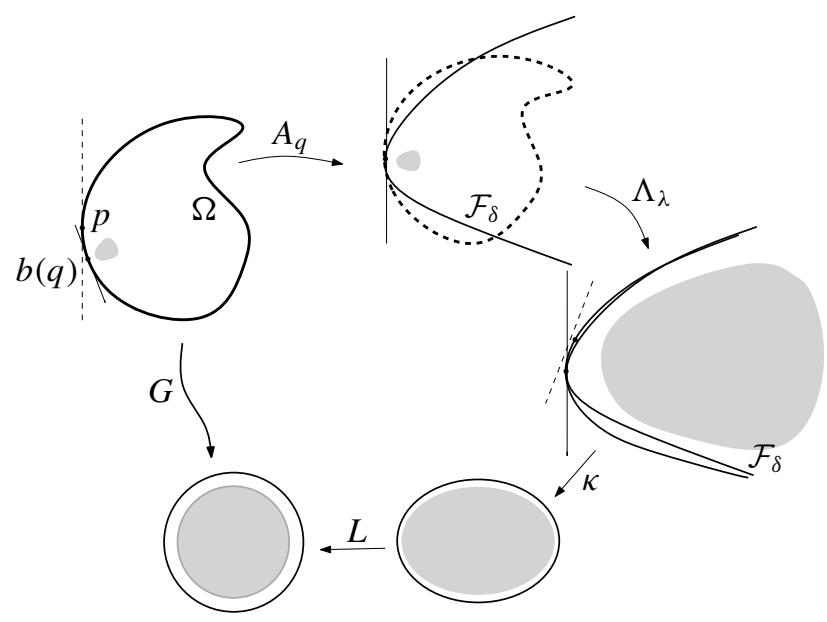

Figure 5. $\mathbb{B}^{n}(\mathbf{0} ; 1-\epsilon) \subset G(\Omega)$.

Now we show that $G(\Omega) \subset \mathbb{B}^{n}(\mathbf{0} ; 1+\epsilon)$. Consider

$$
\Omega^{\prime}:=\Omega-\mathbb{B}^{n}\left(p ; r_{2}\right) .
$$

Notice that there exists an integer $\ell \gg 1$ such that

$$
A_{q}\left(\Omega^{\prime}\right) \subset A_{q}(\Omega)-\mathbb{B}^{n}\left(\mathbf{0} ; r_{2} / \ell\right) \subset \mathcal{E}-\mathbb{B}^{n}\left(\mathbf{0} ; r_{2} / \ell\right) .
$$

Now, there is an integer $m_{4}>m_{3}$ such that, if $m>m_{4}$ and $q \in \mathbb{B}^{n}\left(p ; r_{3} /(2 k(m))\right)$, then

$$
\Lambda_{\lambda}\left(\mathcal{E}-\mathbb{B}^{n}\left(\mathbf{0} ; r_{2} / k\right)\right) \subset\left\{z \in \mathcal{E}: \operatorname{Re} z_{1}>\frac{r_{2}}{r_{3}} \cdot \frac{m_{4}}{\ell}\right\} .
$$

This implies that there exists $m_{4}$ such that

$$
G\left(\Omega^{\prime}\right) \subset L \circ \kappa\left(\left\{z \in \mathcal{E}: \operatorname{Re} z_{1}>\frac{r_{2}}{r_{3}} \cdot \frac{m_{4}}{\ell}\right\}\right) \subset \mathbb{B}^{n}\left(-\mathbf{1} ; \rho\left(m_{4}\right)\right)
$$

for some $\rho(m)$ which approaches zero as $m$ tends to infinity; a direct computation with the Cayley transform and the choice of $L$ (see (3-14)) verify this immediately. Therefore, choosing $m_{4}$ sufficiently large, we arrive at

$$
G\left(\Omega^{\prime}\right) \subset \mathbb{B}^{n}(-\mathbf{1} ; \epsilon)
$$

as in Figure 6. For the $\epsilon$ given above, there exists $\delta$ such that

$$
L \circ \kappa\left(\mathcal{G}_{\delta}\right) \subset \mathbb{B}^{n}(\mathbf{0} ; 1+\epsilon) .
$$

Fix this $\delta$, and recall how the auxiliary domain $\mathcal{G}_{\delta}$ was defined in (3-16). Given any $\delta>0$, according to (3-4)-(3-6), there exists $\rho>0$ such that

$$
A_{q}(\Omega) \cap \mathbb{B}^{n}(\mathbf{0} ; \rho) \subset \mathcal{G}_{\delta} .
$$




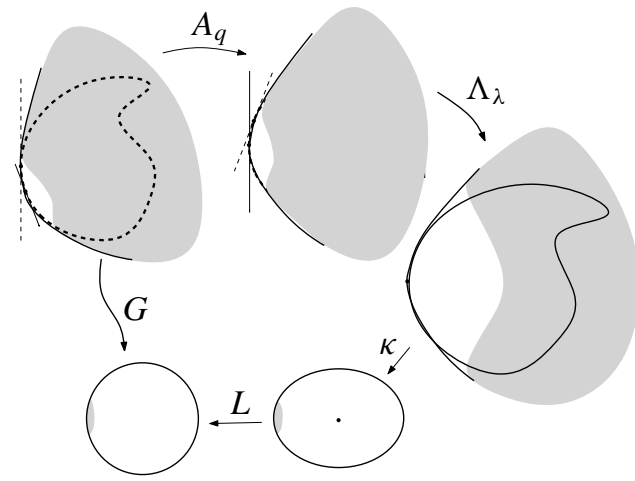

Figure 6. $G\left(\Omega^{\prime}\right) \subset \mathbb{B}^{n}(-1 ; \epsilon)$.

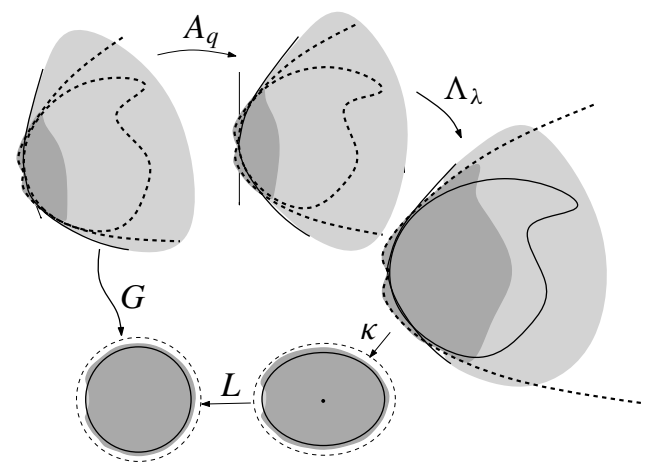

Figure 7. $G(\Omega) \subset \mathbb{B}^{n}(\mathbf{0} ; 1+\epsilon)$.

On the other hand, we can go back to (3-26) and require that $r_{2} / \ell<\rho / 2$. Then we have

$$
A_{q}(\Omega) \cap \mathbb{B}^{n}\left(\mathbf{0} ; 2 r_{2} / \ell\right) \subset \mathcal{G}_{\delta} .
$$

Since there exists an integer $m_{5}>0$ such that $A_{q}\left(\mathbb{B}^{n}\left(p ; r_{2} / \ell\right)\right) \subset \mathbb{B}^{n}\left(\mathbf{0} ; 2 r_{2} / \ell\right)$, we have that

$$
G\left(\Omega-\Omega^{\prime}\right) \subset L \circ \kappa \circ \Lambda_{\lambda}\left(A_{q}(\Omega) \cap \mathbb{B}^{n}\left(\mathbf{0} ; 2 r_{2} / \ell\right)\right) .
$$

This implies

$$
\begin{aligned}
G\left(\Omega-\Omega^{\prime}\right) & \subset L \circ \kappa \circ \Lambda_{\lambda}\left(A_{q}(\Omega) \cap \mathbb{B}^{n}\left(\mathbf{0} ; 2 r_{2} / \ell\right)\right) \\
& \subset L \circ \kappa \circ \Lambda_{\lambda}\left(\mathcal{G}_{\delta}\right) \\
& \subset L \circ \kappa\left(\mathcal{G}_{\delta}\right) \quad \text { by (3-29) } \\
& \subset \mathbb{B}^{n}(\mathbf{0} ; 1+\epsilon) .
\end{aligned}
$$


By (3-27) and (3-30), as in Figure 7 we have that

$$
G(\Omega) \subset \mathbb{B}^{n}(\mathbf{0} ; 1+\epsilon) .
$$

This completes the proof of the claim, and therefore of Theorem 3.1.

\section{Remarks}

In this final section we present several remarks.

On the spherically extreme points. Pertaining to the question in the introduction, one of the naturally arising questions would be whether one may re-embed (the closure of) the bounded strongly pseudoconvex domain so that the preselected boundary point becomes spherically extreme. This question was answered affirmatively in by Diederich, Fornaess, and Wold in [Diederich et al. 2014]. Owing to this new result, Theorem 3.1 now implies the following.

Theorem 4.1. If $\Omega$ is a bounded domain in $\mathbb{C}^{n}$ with a $C^{2}$-smooth strongly pseudoconvex boundary, then $\lim _{\Omega \ni z \rightarrow \partial \Omega} \sigma_{\Omega}(z)=1$.

On the other hand, a more ambitious goal may be to re-embed the domain using the automorphisms of $\mathbb{C}^{n}$ to achieve the same result. But this cannot work, as shown by the following counterexample.

Example. Consider the domain $U$ which is the open 1/10-tubular neighborhood of the circle $S:=\left\{\left(e^{i t}, 0\right) \in \mathbb{C}^{2}: t \in \mathbb{R}\right\}$. This domain is strongly pseudoconvex. Let $p=(9 / 10,0)$. Clearly $p \in \partial U$. If there were $\psi \in \operatorname{Aut}\left(\mathbb{C}^{2}\right)$ which makes $\psi(p)$ spherically extreme for $\psi(U)$, then consider the analytic disc $\Sigma:=\psi(\Delta)$ where $\Delta:=\{(z, 0):|z| \leq 1\})$. Since $\Delta$ crosses $\partial U$ transversally at $\psi(p), \Sigma$ crosses the sphere envelope at $\psi(p)$ and extends to the exterior of the sphere. On the other hand, the boundary of $\Sigma$ remains inside $\psi(U)$ and hence inside the sphere. Now let the sphere expand radially from its center, stopping at the radius beyond which it cannot intersect the holomorphic disc $\Sigma$. Then the sphere is tangent to a point to $\Sigma$ at an interior point, keeping the whole disc inside the sphere. The maximum principle now implies that $\Sigma$ should be entirely on the sphere. But the boundary of $\Sigma$ is strictly inside the sphere, which is a contradiction. This implies that $p$ cannot be made spherically extreme via any re-embedding by an automorphism of $\mathbb{C}^{n}$.

Acknowledgement: This example was obtained after a valuable discussion between Kim and Josip Globevnik. Kim would like to express his thanks to Josip Globevnik for pointing out such a possibility.

On the exhaustion theorem by Fridman-Ma. The main theorem by Buma Fridman and Daowei Ma [1995] obtained the conclusion of Theorem 3.1 in the special case $\Omega \ni q \rightarrow p$ transversely to the boundary $\partial \Omega$. However, that is not sufficient 
to prove Theorem 3.1; it is indeed necessary to consider all possible sequences approaching the boundary. Fridman and Ma [1995] did not need to consider the point sequences approaching the boundary tangentially, as their interest was only on the holomorphic exhaustion of the ball by the biholomorphic images of a bounded strongly pseudoconvex domain. On the other hand, our proof of Theorem 3.1 gives a proof to their theorem as well; we only need to use $(1+\epsilon)^{-1} G(z)$ instead of $G$. (Recall that $G$ depends upon $q$. Letting $q$ converge to $p$ and $\epsilon$ tend to zero, one gets a sequence of maps that exhausts the unit ball holomorphically.)

Plane domain cases. For domains in $\mathbb{C}$, several theorems have been obtained by F. Deng, Q. Guan, and L. Zhang [Deng et al. 2012]. Theorem 3.1 obviously includes many of those results, as every boundary point of a plain domain with $C^{2}$-smooth boundary is spherically extreme.

\section{Acknowledgements}

This research is supported in part by SRC-GaiA (Center for Geometry and its Applications) and the Grant 2011-0030044 from the Ministry of Education. The research of Kim is also supported in part by National Research Foundation Grant 2011-0007831 of South Korea.

\section{References}

[Deng et al. 2012] F. Deng, Q. Guan, and L. Zhang, "Some properties of squeezing functions on bounded domains", Pacific J. Math. 257:2 (2012), 319-341. MR 2972468 Zbl 1254.32015

[Deng et al. 2015] F. Deng, Q. Guan, and L. Zhang, "Properties of squeezing functions and global transformations of bounded domains", Trans. Amer. Math. Soc. (online publication August 2015).

[Diederich et al. 2014] K. Diederich, J. E. Fornæss, and E. F. Wold, "Exposing points on the boundary of a strictly pseudoconvex or a locally convexifiable domain of finite 1-type", J. Geom. Anal. 24:4 (2014), 2124-2134. MR 3261733 Zbl 1312.32006

[Fridman and Ma 1995] B. L. Fridman and D. Ma, "On exhaustion of domains", Indiana Univ. Math. J. 44:2 (1995), 385-395. MR 96h:32028 Zbl 0843.32014

[Kim 1992] K.-T. Kim, "Asymptotic behavior of the curvature of the Bergman metric of the thin domains”, Pacific J. Math. 155:1 (1992), 99-110. MR 93f:32025 Zbl 0723.32009

[Liu et al. 2004] K. Liu, X. Sun, and S.-T. Yau, "Canonical metrics on the moduli space of Riemann surfaces I”, J. Differential Geom. 68:3 (2004), 571-637. MR 2007g:32009 Zbl 1078.30038

[Liu et al. 2005] K. Liu, X. Sun, and S.-T. Yau, "Canonical metrics on the moduli space of Riemann surfaces II”, J. Differential Geom. 69:1 (2005), 163-216. MR 2007g:32010 Zbl 1086.32011

[Pinchuk 1991] S. Pinchuk, "The scaling method and holomorphic mappings", pp. 151-161 in Several complex variables and complex geometry, Part 1 (Santa Cruz, CA, 1989), edited by E. Bedford et al., Proceedings of Symposia in Pure Mathematics 52, American Mathematical Society, Providence, RI, 1991. MR 92i:32031 Zbl 0744.32013

[Yeung 2009] S.-K. Yeung, "Geometry of domains with the uniform squeezing property", Adv. Math. 221:2 (2009), 547-569. MR 2010b:32034 Zbl 1165.32004 
Received October 8, 2014. Revised October 13, 2015.

KANG-TAE KIM

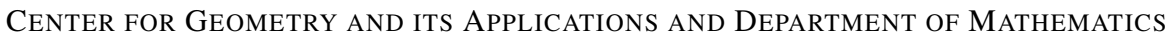
POHANG UNIVERSITY OF SCIENCE AND TECHNOLOGY

POHANG 790-784

SOUTH KOREA

kimkt@postech.ac.kr

LIYOU ZHANG

SCHOOL OF MATHEMATICAL SCIENCE

CAPITAL NORMAL UNIVERSITY

BEIJING, 100048

CHINA

zhangly@cnu.edu.cn 


\title{
PACIFIC JOURNAL OF MATHEMATICS
}

\author{
msp.org/pjm
}

Founded in 1951 by E. F. Beckenbach (1906-1982) and F. Wolf (1904-1989)

\section{EDITORS}

Don Blasius (Managing Editor)

Department of Mathematics

University of California

Los Angeles, CA 90095-1555

blasius@math.ucla.edu

\author{
Paul Balmer \\ Department of Mathematics \\ University of California \\ Los Angeles, CA 90095-1555 \\ balmer@math.ucla.edu \\ Robert Finn \\ Department of Mathematics \\ Stanford University \\ Stanford, CA 94305-2125 \\ finn@math.stanford.edu \\ Sorin Popa \\ Department of Mathematics \\ University of California \\ Los Angeles, CA 90095-1555 \\ popa@math.ucla.edu
}

\author{
Vyjayanthi Chari \\ Department of Mathematics \\ University of California \\ Riverside, CA 92521-0135 \\ chari@math.ucr.edu \\ Kefeng Liu \\ Department of Mathematics \\ University of California \\ Los Angeles, CA 90095-1555 \\ liu@math.ucla.edu \\ Jie Qing \\ Department of Mathematics \\ University of California \\ Santa Cruz, CA 95064 \\ qing@ cats.ucsc.edu
}

\section{PRODUCTION}

Silvio Levy, Scientific Editor, production@msp.org

\section{SUPPORTING INSTITUTIONS}

ACADEMIA SINICA, TAIPEI

CALIFORNIA INST. OF TECHNOLOGY

INST. DE MATEMÁTICA PURA E APLICADA

KEIO UNIVERSITY

MATH. SCIENCES RESEARCH INSTITUTE

NEW MEXICO STATE UNIV.

OREGON STATE UNIV.

\author{
STANFORD UNIVERSITY \\ UNIV. OF BRITISH COLUMBIA \\ UNIV. OF CALIFORNIA, BERKELEY \\ UNIV. OF CALIFORNIA, DAVIS \\ UNIV. OF CALIFORNIA, LOS ANGELES \\ UNIV. OF CALIFORNIA, RIVERSIDE \\ UNIV. OF CALIFORNIA, SAN DIEGO \\ UNIV. OF CALIF., SANTA BARBARA
}

\author{
Daryl Cooper \\ Department of Mathematics \\ University of California \\ Santa Barbara, CA 93106-3080 \\ cooper@math.ucsb.edu \\ Jiang-Hua Lu \\ Department of Mathematics \\ The University of Hong Kong \\ Pokfulam Rd., Hong Kong \\ jhlu@maths.hku.hk \\ Paul Yang \\ Department of Mathematics \\ Princeton University \\ Princeton NJ 08544-1000 \\ yang@math.princeton.edu
}

These supporting institutions contribute to the cost of publication of this Journal, but they are not owners or publishers and have no responsibility for its contents or policies.

See inside back cover or msp.org/pjm for submission instructions.

The subscription price for 2016 is US $\$ 440 /$ year for the electronic version, and $\$ 600 /$ year for print and electronic.

Subscriptions, requests for back issues and changes of subscriber address should be sent to Pacific Journal of Mathematics, P.O. Box 4163, Berkeley, CA 94704-0163, U.S.A. The Pacific Journal of Mathematics is indexed by Mathematical Reviews, Zentralblatt MATH, PASCAL CNRS Index, Referativnyi Zhurnal, Current Mathematical Publications and Web of Knowledge (Science Citation Index).

The Pacific Journal of Mathematics (ISSN 0030-8730) at the University of California, c/o Department of Mathematics, 798 Evans Hall \#3840, Berkeley, CA 94720-3840, is published twelve times a year. Periodical rate postage paid at Berkeley, CA 94704, and additional mailing offices. POSTMASTER: send address changes to Pacific Journal of Mathematics, P.O. Box 4163, Berkeley, CA 94704-0163.

PJM peer review and production are managed by EditFLOW ${ }^{\circledR}$ from Mathematical Sciences Publishers.

\section{PUBLISHED BY}

\section{mathematical sciences publishers \\ nonprofit scientific publishing}

http://msp.org/

(C) 2016 Mathematical Sciences Publishers 


\section{PACIFIC JOURNAL OF MATHEMATICS}

Volume $282 \quad$ No. $2 \quad$ June 2016

Exhausting curve complexes by finite rigid sets

JAVIER ARAMAYONA and CHRISTOPHER J. LEININGER

A variational characterization of flat spaces in dimension three

Giovanni Catino, Paolo Mastrolia and Dario D. Monticelli

Estimates of the gaps between consecutive eigenvalues of Laplacian

293

DAgUang Chen, TAO Zheng and HongCANG YANG

Liouville type theorems for the $p$-harmonic functions on certain manifolds

JINGYI CHEN and YUE WANG

Cartan-Fubini type rigidity of double covering morphisms of quadratic manifolds

\section{HOSUNG KIM}

On the uniform squeezing property of bounded convex domains in $\mathbb{C}^{n}$

KANG-TAE KIM and LIYOU ZHANG

Lefschetz pencils and finitely presented groups

RYOMA KOBAYASHI and NAOYUKI MONDEN

Knot homotopy in subspaces of the 3-sphere

YUYA KODA and MAKOTO OzAWA

On the relationship of continuity and boundary regularity in prescribed mean curvature Dirichlet problems

KIRK E. LANCASTER and JARON MELIN

Bridge spheres for the unknot are topologically minimal

JUNG HOON LEE

On the geometric construction of cohomology classes for cocompact discrete subgroups of $\mathrm{SL}_{n}(\mathbb{R})$ and $\mathrm{SL}_{n}(\mathbb{C})$

SUSANNE SCHIMPF

On Blaschke's conjecture

Xiaole Su, Hongwei Sun and Yusheng Wang

The role of the Jacobi identity in solving the Maurer-Cartan structure equation 